\title{
NEW BOOK ANNOUNCEMENT
}

\section{Collet, P., Martínez, S. And San Martín, J. (2013). Quasi-Stationary Distributions}

is a new volume in the series

\section{Probability and Its Applications}

published by Springer in collaboration with the Applied Probability Trust.

Main concepts of quasi-stationary distributions (QSDs) for killed processes are the focus of the present volume. For diffusions, the killing is at the boundary and for dynamical systems there is a trap. The authors present the QSDs as the ones that allow describing the long-term behavior conditioned to not being killed. Studies in this research area started with Kolmogorov and Yaglom and in the last few decades have received a great deal of attention. The authors provide the exponential distribution property of the killing time for QSDs, present the more general result on their existence and study the process of trajectories that survive forever. For birth-and-death chains and diffusions, the existence of a single or a continuum of QSDs is described. They study the convergence to the extremal QSD and give the classification of the survival process. In this monograph, the authors discuss Gibbs QSDs for symbolic systems and absolutely continuous QSDs for repellers.

The findings described are relevant to researchers in the fields of Markov chains, diffusions, potential theory, dynamical systems, and in areas where extinction is a central concept. The theory is illustrated with numerous examples. The volume uniquely presents the distribution behavior of individuals who survive in a decaying population for a very long time. It also provides the background for applications in mathematical ecology, statistical physics, computer sciences, and economics.

\section{Contents}

1. Introduction

2. Quasi-Stationary Distributions: General Results

3. Markov Chains on Finite Spaces

4. Markov Chains on Countable
Spaces

5. Birth-and-Death Chains

6. Regular Diffusions on $[0, \infty)$

7. Infinity as Entrance Boundary

8. Dynamical Systems 


\title{
NEW BOOK ANNOUNCEMENT
}

\author{
Malyarenko, A. (2013). \\ Invariant Random Fields on Spaces with a Group Action
}

is a new volume in the series

\section{Probability and Its Applications}

published by Springer in collaboration with the Applied Probability Trust.

The author describes the current state of the art in the theory of invariant random fields. This theory is based on several different areas of mathematics, including probability theory, differential geometry, harmonic analysis, and special functions. The present volume unifies many results scattered throughout the mathematical, physical, and engineering literature, as well as it introduces new results from this area first proved by the author. The book also presents many practical applications, in particular in such highly interesting areas as approximation theory, cosmology and earthquake engineering. It is intended for researchers and specialists working in the fields of stochastic processes, statistics, functional analysis, astronomy, and engineering.

\section{Contents}

1. Introduction

2. Spectral Expansions

3. $L^{2}$ Theory of Invariant Random Fields
4. Sample Path Properties of Gaussian Invariant Random Fields

5. Applications 


\section{NEW BOOK ANNOUNCEMENT}

Costa, O. L. V., Fragoso, M. D. AND Todorov, M. G. (2013).
Continuous-Time Markov Jump Linear Systems

is a new volume in the series

\section{Probability and Its Applications}

published by Springer in collaboration with the Applied Probability Trust.

It has been widely recognized nowadays the importance of introducing mathematical models that take into account possible sudden changes in the dynamical behavior of high-integrity systems or a safety-critical system. Such systems can be found in aircraft control, nuclear power stations, robotic manipulator systems, integrated communication networks and large-scale flexible structures for space stations, and are inherently vulnerable to abrupt changes in their structures caused by component or interconnection failures. In this regard, a particularly interesting class of models is the so-called Markov jump linear systems (MJLS), which have been used in numerous applications including robotics, economics and wireless communication. Combining probability and operator theory, the present volume provides a unified and rigorous treatment of recent results in control theory of continuous-time MJLS. This unique approach is of great interest to experts working in the field of linear systems with Markovian jump parameters or in stochastic control. The volume focuses on one of the few cases of stochastic control problems with an actual explicit solution and offers material well-suited to coursework, introducing students to an interesting and active research area.

The book is addressed to researchers working in control and signal processing engineering. Prerequisites include a solid background in classical linear control theory, basic familiarity with continuous-time Markov chains and probability theory, and some elementary knowledge of operator theory.

\section{Contents}

1. Introduction

2. A Few Tools and Notations

3. Mean-Square Stability

4. Quadratic Optimal Control with Complete Observations

5. $\mathrm{H}_{2}$ Optimal Control with Complete Observations
6. Quadratic and $\mathrm{H}_{2}$ Optimal Control with Partial Observations

7. Best Linear Filter with Unknown $(x(t), \theta(t))$

8. $H_{\infty}$ Control

9. Design Techniques

10. Some Numerical Examples 


\section{Subscription rates}

Subscription rates for volume 50 (2013) of Journal of Applied Probability (JAP) are as follows (post free and including online access at http://projecteuclid.org/jap/): US\$326.00; A\$316.00; £206.00 for libraries and institutions; or US\$109.00; A $\$ 106.00$; $£ 69.00$ for individuals belonging to a recognised scientific society. The subscription rates for volume 45 (2013) of Advances in Applied Probability, the companion publication, are the same; if both journals are ordered directly from the Applied Probability office at the same time, the combined price is discounted by 10\%. Please send all enquiries to: Applied Probability Subscriptions, School of Mathematics and Statistics, University of Sheffield, Sheffield S3 7RH, UK (telephone +44 114222 3922; fax +44 114222 3926; email s.c.boyles@ sheffield.ac.uk). Cheques, money orders, etc. should be made payable to 'Applied Probability'. Payment is acceptable in US, Australian or UK currency, or by Visa or Mastercard. We can provide back issue prices on application.

\section{Notes for contributors}

Research papers are published in both Journal of Applied Probability (JAP) and Advances in Applied Probability $(A A P)$, with longer papers typically appearing in $A A P$. However, assignation of papers between the two journals is made by the Editor on an issue-by-issue basis. A submission to Applied Probability is considered as a submission to either journal. In addition, $A A P$ publishes letters specifically related to papers that have appeared in $A A P$ and $J A P$ publishes short communications of a few printed pages in the nature of notes and letters specifically related to papers that have appeared in JAP. Review papers and papers in stochastic geometry and statistical applications are published in AAP.

Fifty offprints of each paper will be provided free, with additional offprints available at cost.

Papers submitted to the Applied Probability journals are considered on the understanding that they have not been published previously and are not under consideration by another publication. Accepted papers will not be published elsewhere without the written permission of the Trust. Submitted papers should be in English. It is the author's responsibility to ensure an acceptable standard of language, and a paper failing to meet this requirement may go back to the author for rewriting before being sent out for review.

Papers should include: (i) a short abstract of 4-10 lines giving a non-mathematical description of the subject matter and results; (ii) a list of keywords detailing the contents; and (iii) a list of classifications, using the 2010 Mathematics Subject Classification scheme (http://www.ams.org/msc/). Letters to the Editor need not include these. To assist authors in writing papers in the Applied Probability style, they may use the LTEX class file aptpub.cls, available from http://www.appliedprobability.org/. Use of this class file is not a condition of submission, but will considerably increase the speed at which papers are processed.

Papers should be submitted as hard copy or as electronic files (with hard copy back-up). All submissions will be acknowledged on receipt and must be accompanied by a covering letter stating the author's postal address and affiliation. Hard copy: Send all submissions to the Applied Probability office in Sheffield, and not to individual editors. Two copies of the paper, at least one of which should be double spaced, should be sent to: Executive Editor, Applied Probability, School of Mathematics and Statistics, University of Sheffield, Sheffield S3 7RH, UK. Electronic submission: Please email a double-spaced PostScript ${ }^{\mathrm{TM}}$ (.ps) or portable document format (.pdf) file, not exceeding $1 \mathrm{Mb}$. The files must be clearly identified by name in a separate covering message. The address for email submissions is japaap@sheffield.ac.uk. Authors should also submit one hard copy to the Executive Editor, as above.

\section{Copyright}

The copyright of all published papers is vested in the Applied Probability Trust. When a paper is accepted for publication, the Trust asks the authors to assign copyright by signing a form in which the terms of copyright are listed. Failure to do this promptly may delay or prevent publication.

Authorisation to photocopy items for internal or personal use, or the internal or personal use of specific clients, is granted by the Applied Probability Trust for libraries and other users registered with the Copyright Clearance Center (CCC) Transactional Reporting Service, provided that the corresponding processing and royalty fees (see http://www.copyright.com) are paid directly to CCC, 222 Rosewood Drive, Danvers, MA 01923, USA. 0021-9002/13 


\section{Volume 50 Number 1}

\section{Research Papers}

1 CHRIS SHERLOCK. Optimal scaling of the random walk Metropolis: general criteria for the 0.234 acceptance rule

16 JOSH REED, AMY WARD AND DONGYUAN ZHAN. On the generalized drift Skorokhod problem in one dimension

29 ALEXANDRA CHRONOPOULOU AND GEORGIOS FELLOURIS. Optimal sequential change detection for fractional diffusion-type processes

42 GIOVANNI PUCCETTI AND LUDGER RÜSCHENDORF. Sharp bounds for sums of dependent risks

54 KEISUKE MATSUMOTO AND TOSHIO NAKATA. Limit theorems for a generalized Feller game

64 DENIS DENISOV AND VSEVOLOD SHNEER. Asymptotics for the first passage times of Lévy processes and random walks

85 JOHAN S. H. VAN LEEUWAARDEN AND KILIAN RASCHEL. Random walks reaching against all odds the other side of the quarter plane

103 KRZYSZTOF DȨBICKI, IWONA SIERPIŃSKA AND BERT ZWART. Asymptotics of hybrid fluid queues with Lévy input

114 HANJUN ZHANG AND YIXIA ZHU. Domain of attraction of the quasistationary distribution for birth-and-death processes

127 BO JIANG, JIAN TAN, WEI WEI, NESS SHROFF AND DON TOWSLEY. Heavy tails in queueing systems: impact of parallelism on tail performance

151 HENDRIK BAUMANN AND WERNER SANDMANN. Computing stationary expectations in level-dependent $\mathrm{QBD}$ processes

166 HOLGER FINK, CLAUDIA KLÜPPELBERG AND MARTINA ZÄHLE. Conditional distributions of processes related to fractional Brownian motion

184 DÉBORAH FERRÉ, LOÏC HERVÉ AND JAMES LEDOUX. Regular perturbation of $V$-geometrically ergodic Markov chains

195 BIN LI, QIHE TANG AND XIAOWEN ZHOU. A time-homogeneous diffusion model with tax

208 AMAURY LAMBERT AND PIETER TRAPMAN. Splitting trees stopped when the first clock rings and Vervaat's transformation

228 KEVIN LECKEY AND RALPH NEININGER. Asymptotic analysis of Hoppe trees

239 SHUHEI MANO. Ancestral graph with bias in gene conversion

256 SHUHEI MANO. Duality between the two-locus Wright-Fisher diffusion model and the ancestral process with recombination

272 M. BURKSCHAT AND J. NAVARRO. Dynamic signatures of coherent systems based on sequential order statistics

\section{Short Communications}

288 OFFER KELLA AND WOLFGANG STADJE. Asymptotic expected number of passages of a random walk through an interval

295 ADAM METZLER. The Laplace transform of hitting times of integrated geometric Brownian motion

300 YI-CHING YAO. A duality relation between the workload and attained waiting time in FCFS $\mathrm{G} / \mathrm{G} / s$ queues 